\title{
FireDST: Fire Impact and Risk Evaluation Decision Support Tool - model description
}

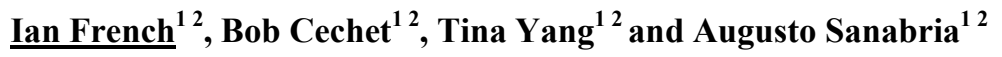 \\ 1. Environmental Geoscience Division, Geoscience Australia, Canberra, 2609 Australia \\ 2. Bushfire Cooperative Research Centre, Melbourne Australia \\ Email:ian.french@ga.gov.au
}

\begin{abstract}
Australia needs to develop and use sophisticated fire modeling techniques as an aid in the prevention and mitigation of bushfires (COAG 2002). This was reinforced by the recommendations from the Victorian Bushfire Royal Commission (VBRC 2010) following the disastrous "Black Saturday" fires of 2009. In 2011, the Australian Bushfire Cooperative Research Centre initiated the FireDST (Fire Impact and Risk Decision Support Tool) research project with a focus on all aspects of modeling extreme fires in Australia including the development of a "proof-of-concept" computer system that could predict the likely probabilistic spread of a bushfire that has already ignited.
\end{abstract}

The FireDST "proof-of-concept" system links various databases and models, including the Australian Bureau of Meteorology's new "high resolution" ACCESS (Australian Community Climate and Earth-System Simulator) numerical weather prediction system, the PHOENIX RapidFire fire spread model, the Commonwealth Scientific and Industrial Research Organisation (CSIRO) building vulnerability assessment model, infrastructure and demographic databases provided by Geoscience Australia and CSIRO's smoke dispersion model is the last stage of the system, and it provides spatial information regarding smoke concentration and likely human impacts. The information is assembled into an integrated simulation framework through a geographical information system (GIS) interface. The Australian Bureau of Statistics (ABS) Census-derived social and economic information as well as extensive quantity surveying of asset values are also included

FireDST is designed to produce probabilistic results that are based on an understanding of the issues that may occur. For example simulating multiple individual fires that have different ignition points and start times, vegetation and fuel characteristics, and variations in wind speed and direction. All the individual simulations are amalgamated into a probabilistic view of the fire spread based on the estimated uncertainties.

This paper provides an overview of the FireDST simulation "proof-of-concept" tool and walks through a sample probabilistic simulation constructed using the tool. The "proof-of-concept" system has demonstrated the successful generation of probabilistic fire spread as well as the impact associated with the probabilistic fire spread. Graphs and tables provide the exposure results (e.g. those houses and people in the 76-100\% probability area).

FireDST is designed to be used to assist in the management of bushfires, land use management (e.g. predicting extent and effectiveness of controlled burns), land planning (such as siting of infrastructure to be less exposed to bushfires) and in education. This paper describes the model in the context of its use in bushfire management by providing an example simulation that emulates the disastrous Kilmore Fire in Victoria on 7 February 2009.

Keywords: $\quad$ bushfire hazard and impact, impact simulation, integrated modeling/simulation 


\section{INTRODUCTION}

The last few decades has seen the development of computerised bushfire spread models that produce a single deterministic simulation of the fire spread. These models include PHOENIX Rapidfire (Tolhurst et al., 2008) in Australia, FireSite (Finney, 1998), FlamMap (Finney, 2006), WFDSS- FSPro (Wildland Fire Decision Support SystemFire Spread Probability Model) (McDaniel, 2007) in the USA, and Prometheus, the Canadian wildland fire growth simulation model (Tymstra et al., 2009). All are able to assimilate information on the terrain, vegetation load and type, built features and weather predictions to produce a single graphical output of the progress of the fire. The models, which are calibrated against how past fires have typically progressed, consider: vegetation type, terrain and topography, a fire's perimeter, air temperature, wind, and humidity. They then model where a fire will go, and when it will arrive at a certain location.

In 2011 the Australian Bushfire Cooperative Research Centre initiated the FireDST research project (Fire Impact and Risk Decision Support Tool). One of the main aims of the project was to develop a "proof-of-concept" system that allows users to easily view and manipulate uncertainties in the information supplied to the fire spread model in order to produce a probabilistic fire spread. The probabilistic fire spread displays many "what-if" scenarios that provide extra essential information to fire managers. FireDST is a bushfire impact and risk simulator that is focused on developing an understanding of the risk to people and infrastructure, with the ultimate aim of providing information which will enable decision-makers to prioritize emergency management resources.

\section{METHODOLOGY}

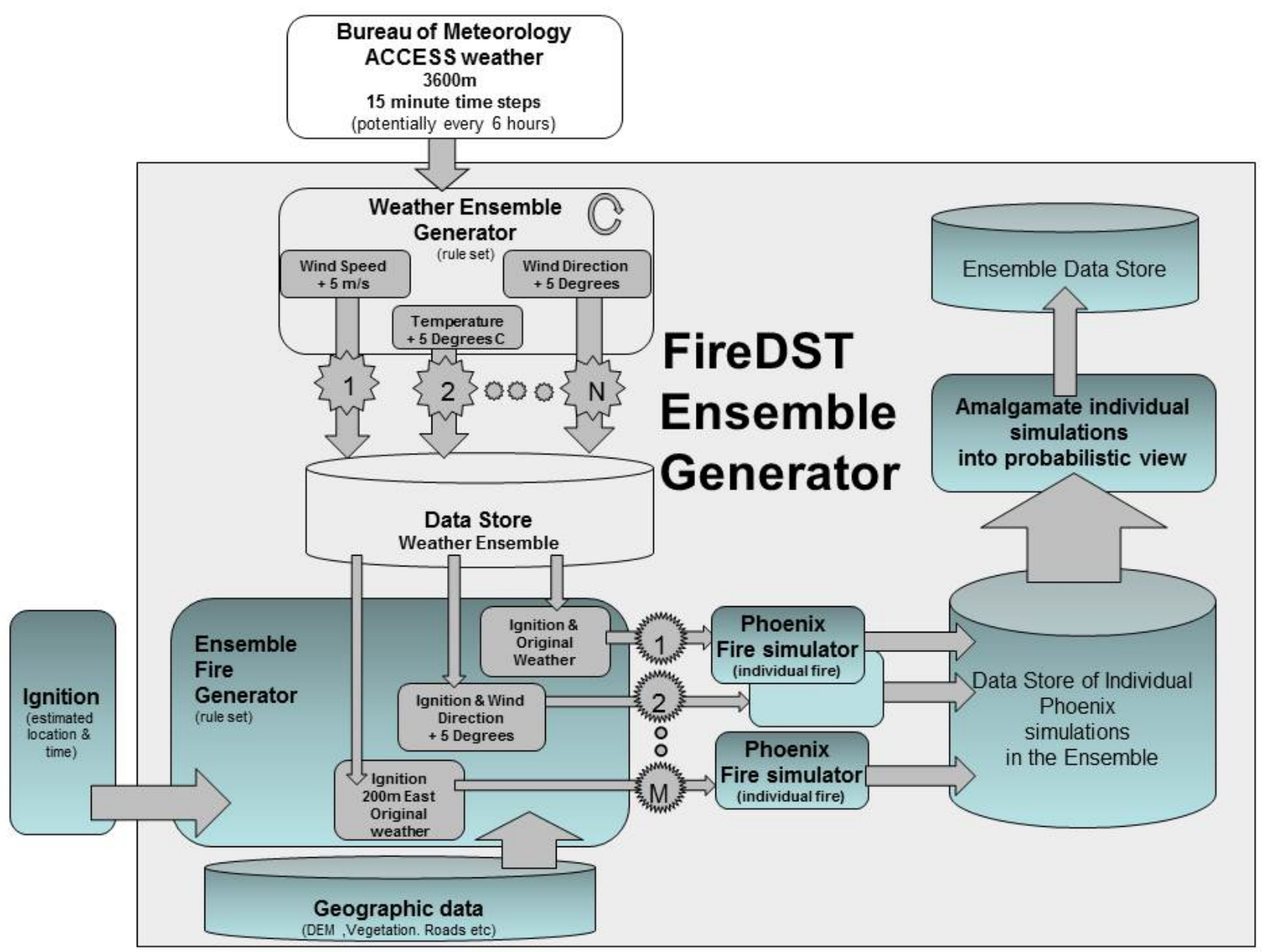

Figure 1. The FireDST Ensemble Generator 
The core of the FireDST system is the Ensemble Generator (Figure 1). The Bureau of Meteorology supplied an ACCESS (Australian Community Climate and Earth-System Simulator)(Puri, 2011) weather simulation prediction for 48 hours at horizontal grid resolutions of $3600 \mathrm{~m}, 1200 \mathrm{~m}$ and $400 \mathrm{~m}$ with 5 minute time steps. Each ACCESS simulation includes separate grids for temperature, relative humidity, wind speed and wind direction at $10 \mathrm{~m}$ height. ACCESS also includes the vertical wind speed/direction described at 50 levels (for the $3600 \mathrm{~m} 15$ minute time step ACCESS only). The Weather Ensemble Generator allows the user to define a set of rules for creating a fixed weather ensemble every time a new ACCESS weather model is produced by the Bureau of Meteorology. The Weather Ensemble Generator modifies the weather grids to produce different combinations of weather that the user considers likely to occur (i.e. takes into account some of the uncertainties in weather conditions). Simple rules are used to modify individual weather scenarios. Examples are changing the wind speed grid to increase all wind speeds by five meters per second, or changing the temperature grid to increase the temperature by two degrees. The rules also allow a more complex weather scenario by creating a profile that includes a combination of changes to humidity, temperature, wind speed or wind direction. These weather variations are stored as a weather ensemble for later use when a fire ignition location is supplied to FireDST.

The Ensemble Fire Generator creates a specific XML input file for each fire simulation in the ensemble, based on a set of user-defined rules. For instance to create a fire ensemble, the user enters the location of an ignition point, ignition time and fire name. FireDST uses the ignition point rules to generate other fires around the user specified ignition point (e.g. 200 metres to the North, South, East and West). The rules can vary the vegetation conditions (such as variations in fuel load and curing) and select a weather ensemble from the weather ensemble generator. The FireDST Ensemble Fire Generator rules also allow the modification of wind speed based on the local terrain/topography using the Wind Ninja system (Forthofer et al., 2009) or wind multipliers (Yang et al., in press). The user also specifies the GIS information that is required by PHOENIX Rapidfire including a vegetation grid, a digital elevation model, road disruptions and fire history database. FireDST uses PHOENIX (supplied by the University of Melbourne) to run each single simulation of the fire.
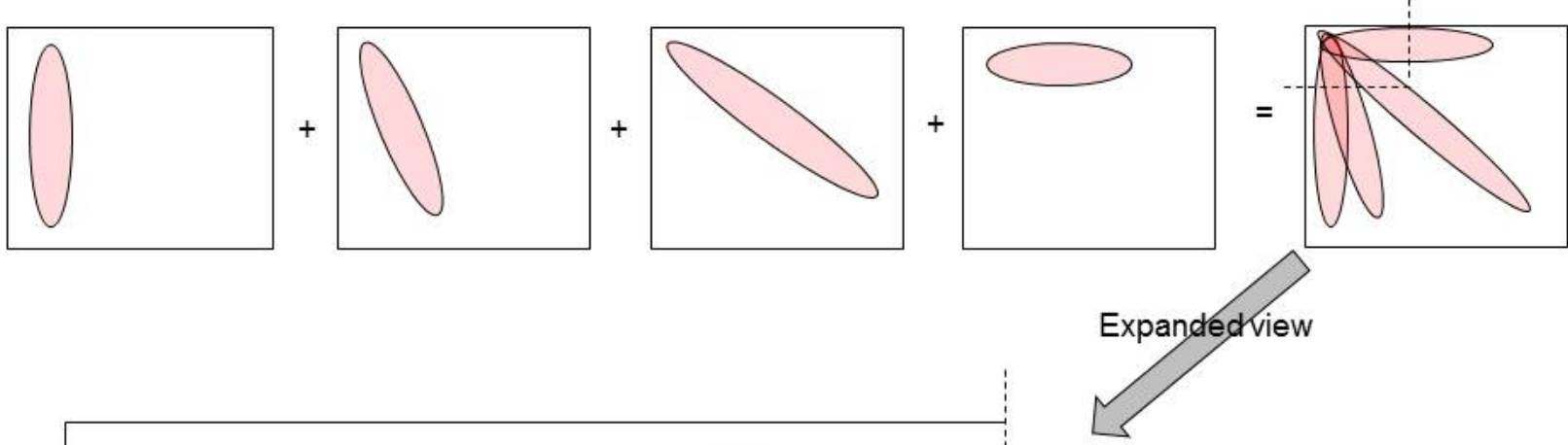

Legend
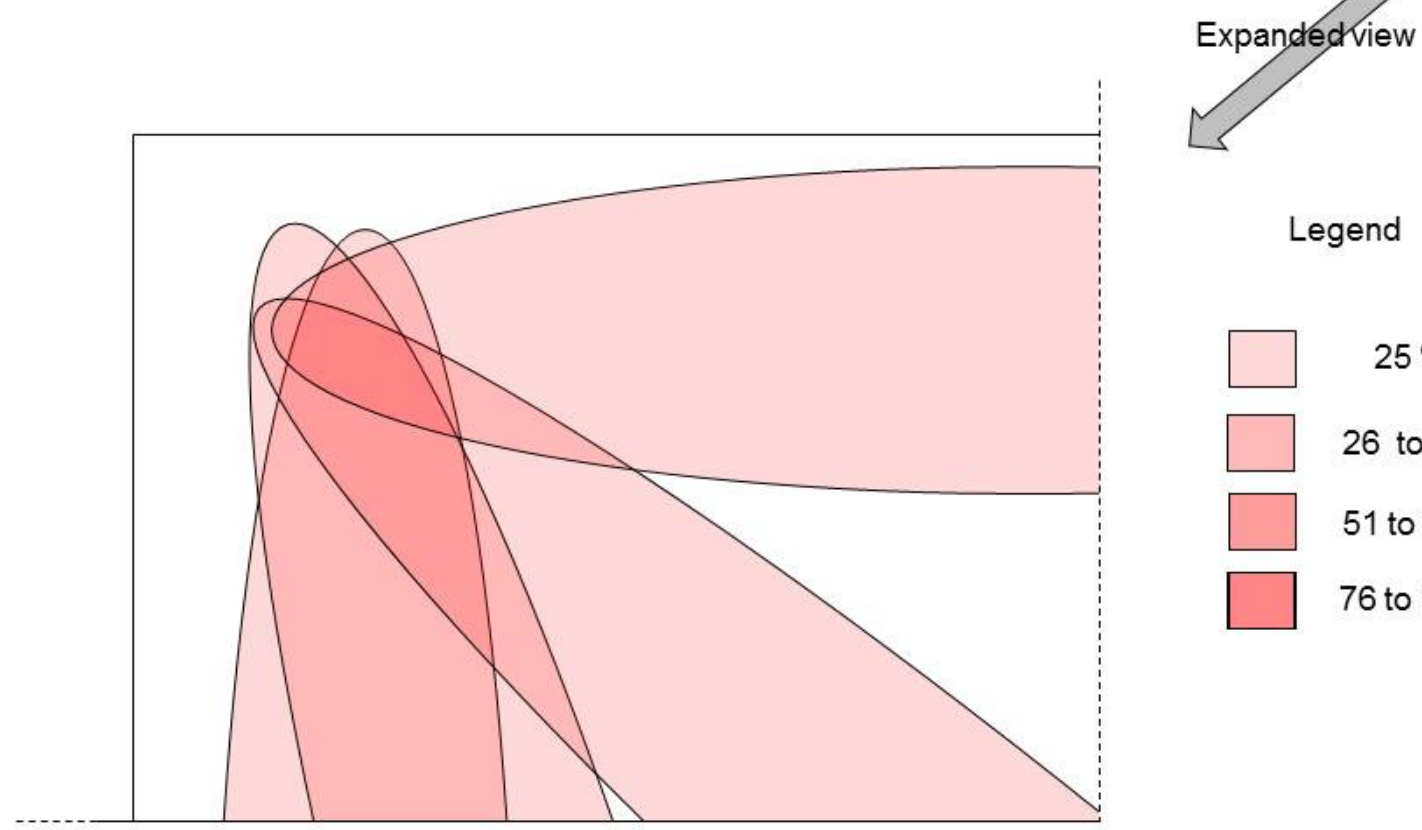

$25 \%$

26 to $50 \%$

51 to $75 \%$

76 to $100 \%$

Figure 2. Example of how a probabilistic shape of a fire is produced. The polygons show the fire spread of individual simulations. 
FireDST produces a probabilistic burn extent from each of the individual simulations in the ensemble by overlaying each of the individual fire spread simulation burn extents with each other. The user would normally select over 30 individual fire simulations to create the probabilistic fire shape. An example of how this is completed is shown in Figure 2 which uses four fire shapes. Initially the internal part of each fire shape is converted to a value of one and everything external to the shape is allocated zero. The shapes are combined and the values in both fires are added. Thus when the first two fire shapes are overlapped you will have areas where both fires have overlapped containing values of two, areas where they did not overlap containing values of one and zero everywhere else. The process is repeated for all the fire shapes in the ensemble. The percentage overlap in the final shape is calculated by converting the numbers in the overlap shape to a percentage of the total number of fires in the ensemble. The percentage shape is then displayed by making ESRI ArcGIS shape files of the groups of percentages required (e.g. increments of $5 \%$, or $10 \%$, or 20\%). The FireDST system can display the probabilistic view at various steps through the simulated timeframe (to identify the fire progression) and at differing percentages of overlap.

Figure 3 shows the complete information flow in FireDST and displays how the FireDST Ensemble Generator results are used. FireDST is able to display statistics and maps of residents and structures exposed to the probabilistic fire spread.

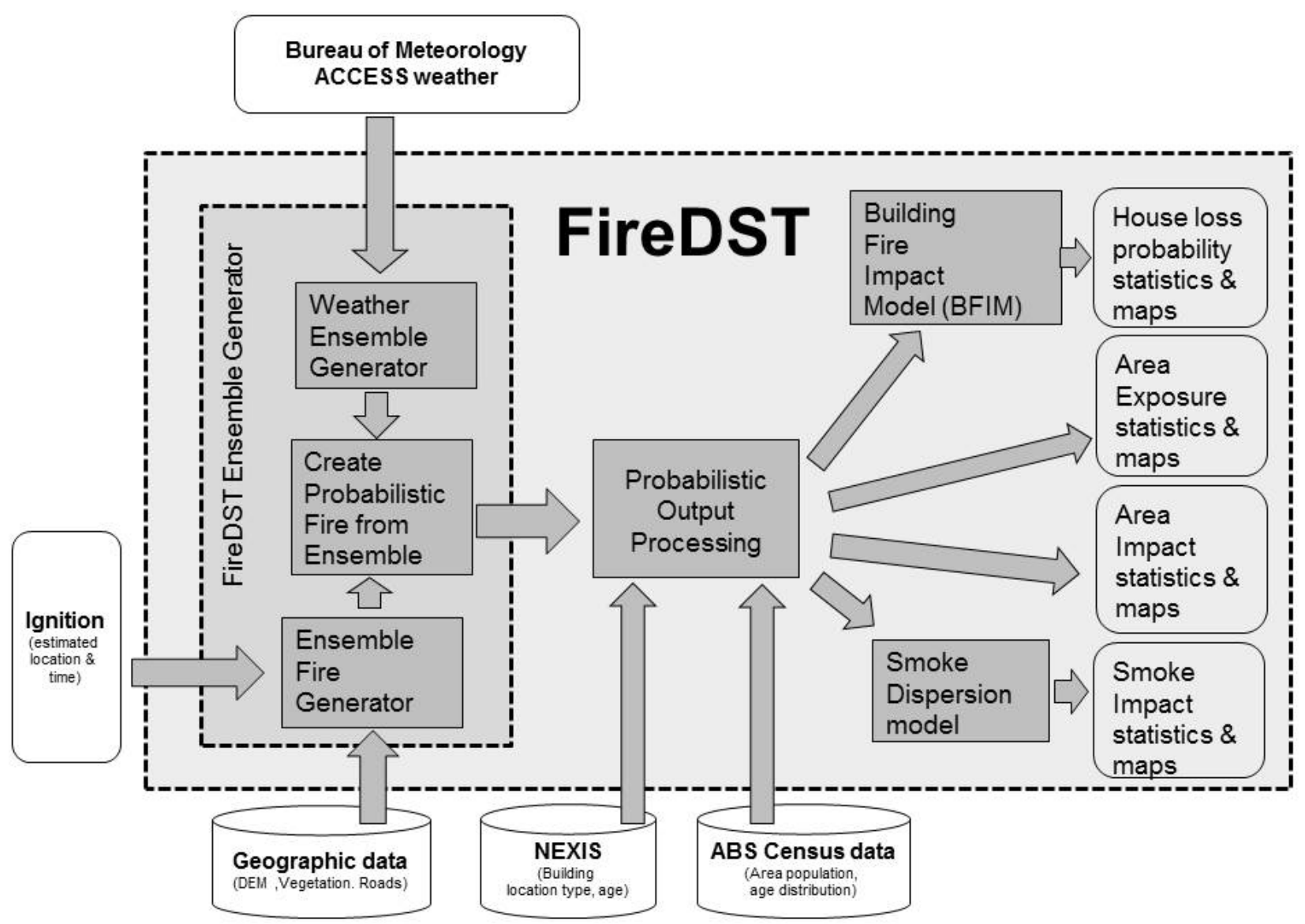

Figure 3. Information flow in FireDST

Geoscience Australia supplied the building database National Exposure Information System (NEXIS) (Nadimpalli, 2007; Canterford, 2011). NEXIS identifies the house location at the driveway entrance (on the road) and so the FireDST team adjusted the house location to match the post-fire Kilmore survey information or located the house at the center of the land parcel. Population statistics were extracted by Geoscience Australia from the 2006 ABS Census (www.abs.gov.au) at the Collection District reference level and allocated on average to all the individual houses in that collection district. FireDST assimilates information about the residence type, occupants and neighbours. FireDST uses the Building Fire Impact Model (BFIM) (Sanabria et al., 2013) to calculate the probability of a house being destroyed by house-to-house fire spread. The BFIM translates the radiation energy and ember density produced by the PHOENIX Rapidfire simulation 
at the 180 metre grid cell where a house exists into the physical interaction (fire impact) with the actual residence. The BFIM considers the response of the structure to the ember density received due to the ember reduction effects of the occupants, their neighbours and any emergency service team that may be present. Conversely, the embers density is increased if the house has been damaged by wind prior to ember attack. The final ember and radiation hazards are then applied to a house (Blanchi et al., 2010) to determine the probability of the house being destroyed by the passing fire. To determine this, FireDST uses a set of generic vulnerability curves supplied by the CSIRO Ecosystem Sciences division. The BFIM is currently a generic model. Future enhancements will involve the interaction of the simulations with the local vegetation and environmental conditions surrounding the building. For this reason the results of the BFIM component of FireDST are more likely to be used in land use planning and mitigation prior to a bushfire rather than as an input to the fire controller when managing a bush fire.

Finally, FireDST's probabilistic output can be passed to a new numerical model that models the atmospheric spread of gaseous fire combustion materials such as smoke, ozone, $\mathrm{PM}_{2.5}$ and $\mathrm{CO}_{2}$ (supplied by CSIRO Marine \& Atmospheric Research). Information regarding this model can be found in Myer et al. (in press). Outputs of the model are maps of the concentration of the various products of bushfire combustion as well as the impacted population. These maps can be useful to assist in managing public health warnings and in the movement of people and emergency service teams.

\section{EXAMPLE}

In this paper we use the example of the Victorian "Black Saturday" Kilmore fire that started at approximately 11:45am on 7 February 2009 (Gellie et al., 2012) and was one of the most disastrous bushfires to have occurred in Australia. It resulted in the loss of 159 lives, destruction of 1639 houses and burning of 255,500 ha of land.

In this example the Weather Ensemble Generator provided a thirty member scenario ensemble which included the following individual fires as a description of the perturbations applied:

- weather supplied by ACCESS model (3600m horizontal resolution; 15 minutes time steps) starting at 11 am on 6 February 2009 for 48 hours;

- wind direction $(\mathrm{Wd})+/-10$ degrees;

- wind speed (Ws) - $+/-5 \mathrm{~m} / \mathrm{s}$;

- air temperature $(\mathrm{T})-2$ degrees $\mathrm{C},+5$ degrees $\mathrm{C}$;

- relative humidity $(\mathrm{RH})$ - decreased by 2 percent $\&$ increased by 5 percent;

- Twenty one combinations of the above values for Wind Direction, Wind Speed, Temperature and Relative Humidity (i.e. T increased by 5 degrees, RH decreased by 2 percent, Wind Speed increased by $5 \mathrm{~m} / \mathrm{s} \&$ Wind Direction increased by +10 degrees).

The Ensemble Fire Generator rules included:

- moving the ignition location to 200 metres to the north, south, east and west of the Kilmore ignition point

- using the Wind Ninja wind modifiers

- no suppression

- maximum fuel load

The vegetation supplied to PHOENIX is the 2009 vegetation model for Victoria at 25 metre resolution and 10 metre Digital Elevation Model (DEM) were both supplied by Victoria’s Department of Sustainability \& Environment.

Figure 4 shows the ensemble probabilistic extent that was generated by overlapping all of the individual fire extents at 1600. The red colour depicts where the individual fire shapes have overlapped for $75-100 \%$ of the simulations; the yellow colour displays where fire spread shapes have overlapped for $3 \%$ to $25 \%$ of the simulations.

Superimposed in Figure 4 is the Kilmore fire reconstruction isochrone for 1600 (Gellie et al., 2012). Ideally the real fire isochrone (or any actual field intelligence about the fire location) should be fully inside the probabilistic shape. Comparison of actual values and the probabilistic shape allows the FireDST user to make decisions about which simulations should be used in the probabilistic fire spread.

FireDST can also display a variety of exposure and impact statistics for the fire that have been extracted from the 2006 ABS Census data displayed at the mesh block level. Figure 4 also shows small bar graphs of the population exposed to the FireDST fire simulation. The graphs show the mesh block statistics for the total population, the population aged over 65 , under 5 and those who marked on the Census that they need assistance. 


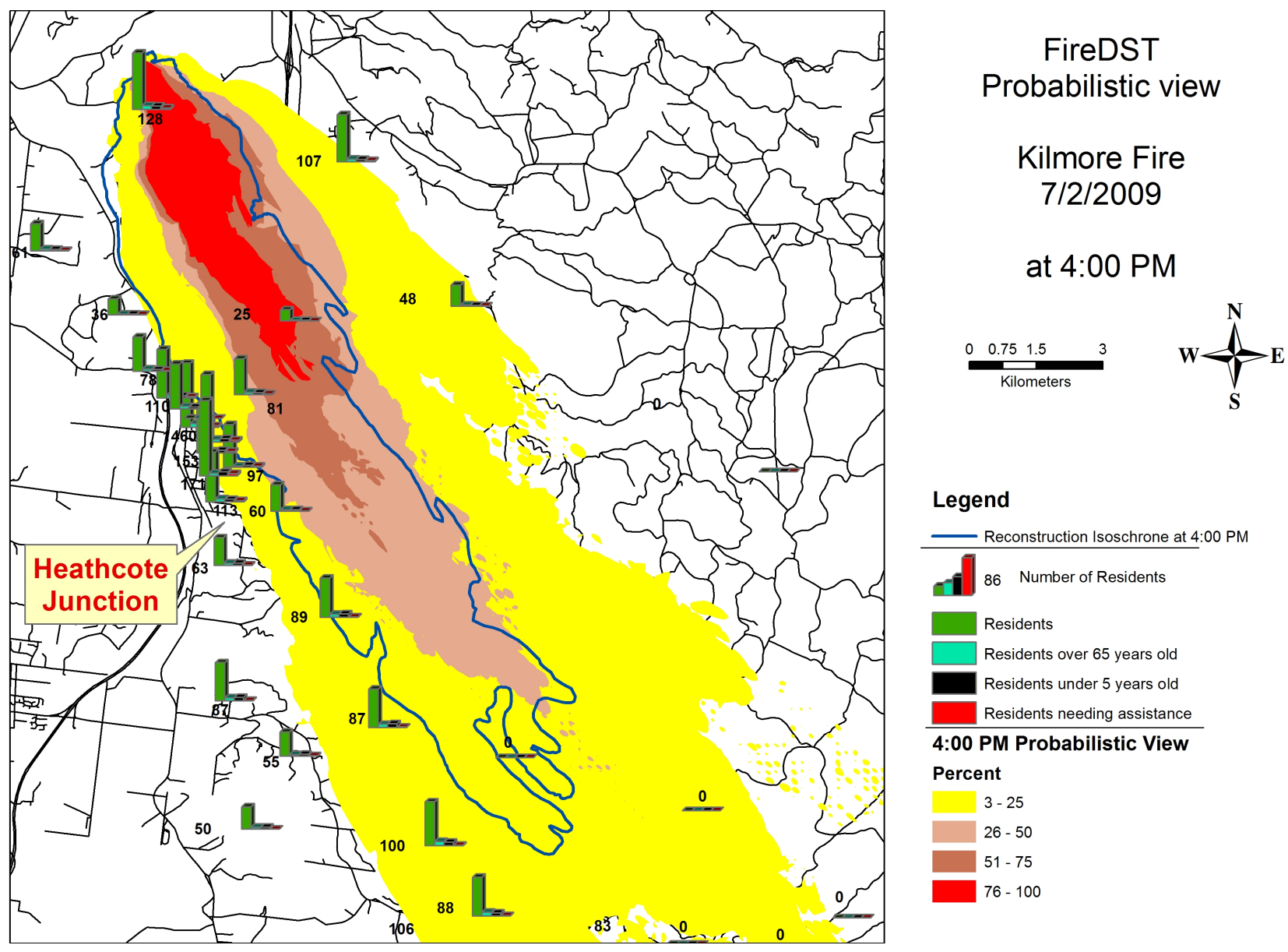

Figure 4. Probabilistic view of the Kilmore fire utilising 30 simulations (weather scenarios) out to four hours and fifteen minutes after ignition; the historical fire reconstruction is shown as the blue line.

\section{DISCUSSION AND CONCLUSION}

The FireDST "proof-of-concept" system has demonstrated the successful generation of probabilistic fire spread as well as modeling the associated impact. FireDST has shown that more than one probabilistic fire spread can be generated that is larger than the actual fire spread for the Kilmore fire. FireDST has also demonstrated the ability to derive and display statistical information about people and buildings that are exposed to the probabilistic results. Graphs and tables can be displayed showing the exposure across the probability extent (e.g. an estimate of those houses and people in the 76-100\% probability area for the simulations considered). Furthermore these statistics can also be produced for each simulation member within the FireDST ensemble, allowing emergency managers to consider impacts with regards to resource deployments.

FireDST could be used in a number of ways by fire and land management agencies. The most significant advancement made is the ability to simulate the probability of impact of a bushfire. The simulation system provides rich information about the potential losses, and how likely they are to occur given ignition, terrain, weather and fuel condition/types. In operational response mode, resource allocation and community warning of a fire and smoke impact can be informed by the probabilistic extent.

The components of the FireDST system, with minor modifications, could also be used for planning applications such as adapting existing buildings through strengthening construction regulations, and then re-simulating to examine the changed impact. Scenario analysis can be used to assess the potential of vegetation modification to mitigate bushfire spread. Likewise "what-if" scenarios could use FireDST in land use planning where various town designs can be examined to assess community resilience to bushfires. FireDST could also be used for educational purposes as part of incident management training and community safety initiatives, where agencies demonstrate to staff, volunteers and the community which homes would be defendable under different conditions. From this, appropriate bushfire survival plans could be made, and the impacts that preparations such as clearing trees or ember-proofing can be demonstrated to the community. 


\section{FUTURE DIRECTION}

The FireDST "proof-of-concept" tool represents significant advances in each of the key areas investigated (weather, probabilistic fire spread, building fire analysis, exposure, impact statistics and smoke modeling). The inclusion of this research into operational emergency management systems is a priority as emergency managers require a robust and reliable simulation system that provides an operational understanding of uncertainties as bushfires develop.

\section{ACKNOWLEDGEMENTS}

This study was partly supported by the Bushfire Cooperative Research Centre as part of the "Understanding Risk" theme. The authors acknowledge the efforts of Jeff Kepert, Kevin Tory, Kevin Tolhurst and Mick Meyer in contributing to the underpinning knowledge and datasets that form some of the modules of FireDST, as well for providing advice/feedback regarding the methodology and products.

\section{REFERENCES}

Blanchi, R., C. Lucas, J. Leonard, and K. Finkele (2010). Meteorological conditions and wildfire-related house loss in Australia. International Journal of Wildland Fire, 19, 7, 914-926

Canterford,S. (2011). Locating People Spatially:2006,2010,2100 and 2:36PM on Friday, Australasian Journal of Regional Studies, (17) $1,46-59$

COAG - Council of Australian Governments (2002). Review of natural disaster relief and mitigation arrangements. Natural disasters in Australia: Reforming mitigation, relief and recovery arrangements. Department of Transport and Regional Services, Canberra

Finney, M. A. (1998). FARSITE: Fire Area Simulation - Model development and evaluation. USDA Forest Service, Rocky Mountain Research Station, Research Paper RMRS-4.

Finney, M.A. (2006). An overview of FlamMap modeling capabilities. In: P.L. Andrews, B.W. Butler (comps.). Fuels ManagementHow to measure success: Conference Proceedings. RMRS-P-41. p 213-219.

Forthofer, J., Shannon, K., and Butler, B. (2009). Simulating diurnally driven slope winds with Wind Ninja. Proc. 8th Symposium on Fire and Forest Meteorology, October 13-15; Kalispell, MT

Gellie, N., Gibos, K., Mattingley, G.,Wells, T., Salkin, O. (2012). Reconstruction of the spread and behaviour of the Black Saturday fires, $7^{\text {th }}$ February 2009, Department of Sustainability and Environment, Government of Victoria, Draft Version 3.3

Jones, T., Woolf, M., Cechet, R.P. and French, I. (2012). Quantitative bushfire risk assessment framework for severe and extreme fires, Australian Meteorological and Oceanographic Journal (62), 171-178

McDaniel, J. (2007). WFDSS: Taking Decision Support into the 21st Century, Wildland Fire Lessons Learnt Center, http://www.wildfirelessons.net/Additional.aspx?Page $=96$

Meyer, C.P.. Lee, S. and Cope, M. (2013). Smoke impacts from prescribed burning in Victoria; developing a risk climatology. Proceedings of the $20^{\text {th }}$ International Congress on Modelling and Simulation (MODSIM 2013), Adelaide, December 2- $6^{\text {th }}$

Nadimpalli, K. Edwards, M. and D. Mullaly (2007). National EXposure Information System (NEXIS) for Australia: Risk Assessment Opportunities (MODSIM 2007; International Congress on Modelling and Simulation. Oxley, L. and Kulasiri, D. (Eds)) 1674-1680

Nadimpalli, K. (2009). National exposure Information System (NEXIS) - A Capability for Evidence Based disaster Management, 7th FIG Regional Conference Spatial Data Serving People: Land Governance and the Environment - Building the Capacity Hanoi, Vietnam, 19-22 October

Puri, K. (2011). “Overview of ACCESS”, http://www.cawcr.gov.au/bmrc/basic/wksp18/papers/Puri.pdf

Sanabria L.A., French I. and Cechet R.P. (2013). Building Fire Impact Model, Proceedings of the $20^{\text {th }}$ International Congress on Modelling and Simulation (MODSIM 2013), Adelaide, December 2-6 ${ }^{\text {th }}$

Tolhurst, K.G., Shields, B.J. and Chong, D.M. (2008). PHOENIX: development and application of a bushfire risk management tool. Australian Journal of Emergency Management, 23(4), 47-54

Tymstra, C.; Bryce, R.W.; Wotton, B.M.; Armitage, O.B. (2009). Development and structure of Prometheus: the Canadian wildland fire growth simulation model. Inf. Rep. NOR-X-417. Nat. Resour. Can., Can. For. Serv., North. For. Cent., Edmonton, AB. . PROMETHEUS

VBRC (2010). The 2009 Victorian Bushfires Royal Commission final report. Summary and four volume report tabled July $31^{\text {st. }}$, available at http://www.royalcommission.vic.gov.au/Commission-Reports

Yang, T., Nadimpalli, K., and Cechet, R. P. (in prep). Wind Multipliers: Computation methodology for local wind hazard assessment. Geoscience Australia Record. GeoCat \# 75299 